\title{
Lidar cross-sections of soot fractal aggregates: Assessment of equivalent-sphere models
}

\author{
Romain Ceolato ${ }^{\mathrm{a}, *}$, Florian Gaudfrin ${ }^{\mathrm{a}, \mathrm{d}}$, Olivier Pujol ${ }^{\mathrm{b}}$, Nicolas Riviere ${ }^{\mathrm{a}}$, Matthew J. Berg ${ }^{\mathrm{c}}$, \\ Christopher M. Sorensen ${ }^{\mathrm{C}}$ \\ a ONERA, The French Aerospace Lab, Toulouse FR 31055, France \\ ${ }^{\mathrm{b}}$ Université de Lille, Laboratoire d'optique atmosphérique, Villeneuve d'Ascq 59655, France \\ ' Department of Physics, Kansas State University, 1228 N. 17th St., Manhattan KS 66506, USA \\ ${ }^{\mathrm{d}}$ LEUKOS, Limoges 87280, France
}

\section{A R T I C L E I N F O}

\section{Article history:}

Received 27 July 2017

Revised 16 October 2017

Accepted 4 December 2017

Available online 6 December 2017

\section{Keywords:}

Lidar

Cross-sections

Equivalent spherical model

Equivalent-sphere approximation

Light-scattering

Soot fractal aggregates

\begin{abstract}
A B S T R A C T
This work assesses the ability of equivalent-sphere models to reproduce the optical properties of soot aggregates relevant for lidar remote sensing, i.e. the backscattering and extinction cross sections. Lidar cross-sections are computed with a spectral discrete dipole approximation model over the visible-toinfrared $(400-5000 \mathrm{~nm})$ spectrum and compared with equivalent-sphere approximations. It is shown that the equivalent-sphere approximation, applied to fractal aggregates, has a limited ability to calculate such cross-sections well. The approximation should thus be used with caution for the computation of broadband lidar cross-sections, especially backscattering, at small and intermediate wavelengths (e.g. UV to visible).
\end{abstract}

(c) 2017 Elsevier Ltd. All rights reserved.

\section{Introduction}

Soot, or black carbon, has received increasing attention in recent years by the scientific [1] and industrial communities [2,3]. Carbonaceous particles result from incomplete combustion and have natural and anthropogenic origins (e.g. urban fires, biomass fires, fossil combustion) and can be found up to the upper troposphere and lower stratosphere. Freshly produced soot are relatively small, i.e. with characteristic size $<50 \mathrm{~nm}$, and consist mainly of quasi-spherical monomers. With time, the monomers aggregate to form complex microscopic clusters with highly-complicated shape and fractal properties [4-7]. These clusters are referred to as soot fractal-like aggregates (SFA) and the impact of fractal morphology has been investigated in numerous recent studies [8,9].

SFA may impact the global radiative budget by scattering and absorbing solar and the terrestrial radiation (the direct effect), and by influencing cloud formation (the indirect effect) [10,11]. For instance, they seem to act as ice nuclei and so play an important role in contrail formation $[12,13]$. This is of scientific interest in the context of climate change with respect to anthropic impact since ice clouds have a strong greenhouse effect [14]. The under-

\footnotetext{
* Correponding author.

E-mail address: romain.ceolato@onera.fr (R. Ceolato).
}

standing of the ice-forming activity of soot particles is closely related to the knowledge of their microphysical properties, which include size, shape, and composition [15]. These airborne contaminants may also have effects on health [16-18].

Lidar methods have been used for several decades to probe radiative properties of the atmosphere through elastic or inelastic scattering $[19,20]$. Atmospheric elastic lidar consists of a collimated pulsed light-source (laser) and a receiver (telescope) and associated optical sensors. Although most lidar systems use a single wavelength, or frequency, laser, multi-wavelength and broadband lasers in lidar have proven to be robust and efficient techniques to characterize and classify different types of aerosols at distance [21-25]. All these techniques use the advantages of multi-spectral information to enhance the retrieval lidar techniques and are based on the single-scattering elastic lidar equation $[26,27]$. This equation is derived from the standard radar equation and provides the power received by the instrument from backscattering at a range $r$ $[\mathrm{m}]$ and for wavelength $\lambda$ expressed as:

$\left.P(r, \lambda)=P_{0}(\lambda) \eta(\lambda) \frac{A_{e f f}}{r^{2}} O(r, \lambda) \beta(r, \lambda) \exp \left(-2 \int_{0}^{r} \alpha\left(r^{\prime}, \lambda\right) d r^{\prime}\right]\right)$

where $P_{0}(\lambda)$ is the power emitted by the laser, $A_{\text {eff }}$ the telescope's effective receiving area, $O(r, \lambda)$ the overlap function, and $\eta(\lambda)$ the optical efficiency. The total aerosol and molecular spectral volume- 
extinction and backscattering coefficients are, respectively, $\alpha(r, \lambda)$ $\left[\mathrm{m}^{-1}\right]$ and $\beta(r, \lambda)\left[\mathrm{m}^{-1} \mathrm{sr}^{-1}\right]$. These volume coefficients depend upon the concentration and cross-sections of the particles in the particulate media. Freshly emitted soot monomers are small compared to the wavelength of light, and thus, not efficient scatterers. However, the size of soot aggregates is close to the wavelength, resulting in more efficient scattering, including backscattering. This makes lidar techniques good candidates for probing soot emissions in the atmosphere [28-30]. The retrieval of microphysical parameters of soot particles from lidar signals is a complex scientific problem as it involves, for instance, extensive knowledge of the soot formation, optics, and chemistry. Various assumptions are often made due to this complexity, in particular: (i) the soot shape is approximated to be an equivalent-sphere; (ii) the soot complex refractive index is assumed known; and (iii) multiple scattering are neglected.

This paper focuses on the first assumption and aims to assess the ability of different sphere-equivalent approximations to reproduce the extinction and backscattering cross sections of soot aggregates relevant to lidar remote sensing. Previous studies have shown the limited performance of equivalent-sphere approximations to calculate the radiative properties of soot or other complex shaped particles like ice crystals [31]. For instance, recent work [32] compares the scattering amplitudes (and phase functions) of four equivalent-spheres to small-aggregate soot particles at two wavelengths (628 and $1100 \mathrm{~nm}$ ) using the generalized multi-particle Mie method and have concluded large errors exist. In the framework of atmospheric lidar, it is thus crucial for retrieval methods to assess the validity of the equivalent-sphere approximation for complex shaped particles such as soot fractal aggregates. Here, relationships between the fractal model and the different approximations are given. Then, a spectral discrete dipole approximation (SDDA) model is used to compute extinction and backscattering cross-sections over a broadband spectrum [33]. For the first time, this paper addresses the use of equivalentsphere approximation in the framework of atmospheric broadband or multispectral lidar and SFA.

\section{Methods and results}

\subsection{Fractal morphology of soot aggregates}

A major issue in calculating the radiative properties of soot arises from the limitless number of soot aggregate shapes. This calls for a fractal approach to model such complex geometry. The morphology of SFAs is usually characterized by the fractal pre-factor $\left(k_{f}\right)$, the fractal dimension $\left(D_{f}\right)$, the number $(N)$ of monomers, and their mean monomer radius $\left(R_{m}\right)$. These quantities are defined, respectively, by the scaling relation [34] as follows:

$N=k_{f}\left(\frac{R_{g}}{R_{m}}\right)^{D_{f}}$

where the radius of gyration $\left(R_{g}\right)$ is defined from $r_{i}$, the distance of the $i$ th monomer from the cluster's center-of-mass:

$R_{g}=\left(\frac{1}{N} \sum_{i=0}^{N} r_{i}^{2}\right)^{\frac{1}{2}}$

Two types of freshly emitted SFAs are investigated below, referred to as chain-like and compact aggregates. These have fractal geometry and are simulated by diffusion-limited clusteraggregation (DLCA) [35-37]. Here, $D_{f}=1.8$ and 2.39 for the chainlike and the compact aggregates, respectively. The monomers, which have $R_{m}=20 \mathrm{~nm}$, have point-contact with other monomers such that overlapping, necking, or sintering (bridges between monomers) are neglected as pictured in Table 1.

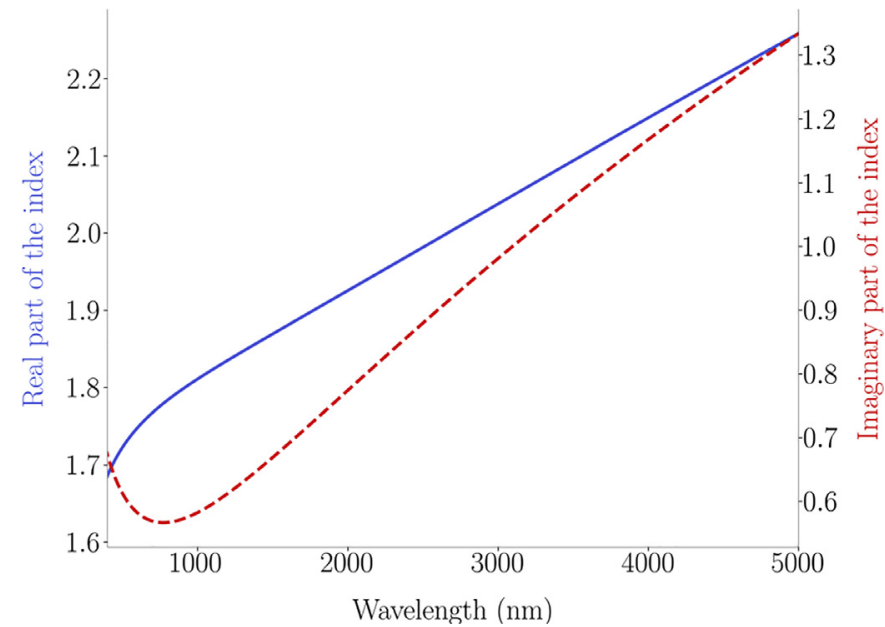

Fig. 1. Spectral complex refractive index $\boldsymbol{m}(\lambda)$ is represented by continuous line (blue, solid curve) for the real part and by dash lines (red, dashed curve) for the imaginary part [41].

\subsection{Spectral discrete dipole approximation}

The method used to calculate the extinction and backscattering cross-sections of SFAs is the coupled-dipole method or discrete dipole approximation (DDA) [38-40]. The SDDA model, an adaptation of the DDA, was developed to compute all the spectropolarimetric light-scattering properties over a wide spectrum ranging from the visible $(400 \mathrm{~nm})$ to the infrared $(5000 \mathrm{~nm})$ [33]. The model simulates a complex-shaped particle, such as a fractal aggregate of spherical monomers, by a finite cubic lattice of interacting electric dipole moments whose polarizability is modeled by the lattice dispersion relation (LDR) for a random orientation. The dipoles residing outside of the aggregate are set to zero. The total electromagnetic field at each electric dipole includes the incident field but also the scattered fields by other surrounding dipoles. The SDDA model is applied to the DLCA-simulated SFAs described above as follows.

The complex refractive index of soot depends on the thermodynamic conditions during formation (pressure, temperature, humidity) and on the chemical composition, which is mainly determined by the type of combustion and fuel (e.g. diesel, biofuel). To perform broadband calculations, a dispersion law is used to model the spectral complex refractive index $m(\lambda)$, given in Fig. 1 [41]. The real and imaginary part of $m(\lambda)=n(\lambda)+i \kappa(\lambda)$ of soot is represented in Fig. 1.

The accuracy of the SDDA model is determined by the fineness of the discretization lattice, i.e., the dipole-dipole spacing, $d$, should be smaller than the wavelength $\lambda$ in the material [39]. Draine provides the criterion $m k d<1$ where $k$ is the wavenumber $k=2 \pi / \lambda$ [42]. For extinction calculations, this criterion was evaluated and mkd was found to be smaller than unity at each wavelength within the broadband spectrum. Moreover, the SDDA model is especially sensitive to the dipole-dipole spacing for scattered intensity into the backscattering direction. For backscattering calculations, the dipole-dipole spacing $d$ should be decreased to become smaller than the spacing required for extinction. The backscattered intensity from SDDA for a sphere was compared to Lorenz-Mie theory to adapt the dipole-dipole spacing for backscattering. The estimated maximum value of the spectral error is below 5\% with the SDDA model for a sphere with the complex refractive index of soot. 
Table 1

Structural parameters and aggregate morphology for the chain-like and compact soot aggregates.

\begin{tabular}{llll}
\hline & Chain-like aggregates & Compact aggregates \\
\hline Structural parameters & $D_{f}$ & 1.8 & 2.39 \\
& $N$ & 90 & 4275 \\
Aggregate morphology & $R_{m}$ & $20 \mathrm{~nm}$ & $20 \mathrm{~nm}$ \\
& & &
\end{tabular}

\subsection{Broadband lidar-relevant parameters}

The spectral extinction cross-sections $C_{\text {ext }}\left[\mathrm{nm}^{2}\right]$ and spectral backscattering cross-sections $C_{\text {back }}\left[\mathrm{nm}^{2} \mathrm{sr}^{-1}\right]$ are computed using the SDDA model for chain-like and compact aggregates over the broadband spectral range of $400-5000 \mathrm{~nm}$. The calculations are carried out with a total of 500 wavelength spread evenly over the spectral range.

In Fig. 2, the extinction and backscattering cross-sections increase with the number of monomers and decrease with increasing the wavelength. This is consistent with previously reported values for the differential scattering cross-section [32]. Moreover, the extinction and differential scattering cross-section are known to be proportional of the number of monomers per aggregate from the Rayleigh-scattering model developed for fractal aggregates [43]. Also note that both cross-sections of chain-like and compact aggregates display an overall decrease with increasing wavelength. Regarding the extinction, the values of the broadband $C_{\text {ext }}$ for the two aggregates present a ratio close to 47.5 which corresponds to the ratio between the monomers number for compact and chainlike aggregates. For the backscattering, the values of the broadband $C_{\text {back }}$ for the two aggregates present a ratio close to $47.5^{2}$, which corresponds to the squared ratio between the monomers defined above. Our results indicate that the extinction cross-sections are directly proportional to the $N$ whereas the backscattering crosssections is proportional to $N^{2}$.

\section{Assessment of equivalent-spherical model}

This section assesses the ability of different equivalent-sphere models to reproduce the broadband backscattering and extinction cross sections for SFAs.

\subsection{Definitions}

The use of equivalent-spheres for optical properties is common practice in remote sensing because spheres both have the simplest geometry and well-known optical properties, i.e. scattering by spheres is solved analytically as Mie theory. When a nonspherical particle is modeled as a spherical particle, only two of the relevant physical parameters (the radius and complex refractive index) are used in the Mie theory surrogate. This simple approximation, referred to as either the equivalent-sphere model or the equivalent-sphere approximation shows several limitations. Although equivalent-spheres can reproduce the radiative properties of ice crystal cloud in the infrared, some care is needed when considering backscattering directions, which is highly dependent on
Table 2

Equivalent sphere radii for volume, surface, and gyration approximation respectively.

\begin{tabular}{llll}
\hline & & Chain-like aggregate & Compact aggregate \\
\hline \multirow{2}{*}{ Equivalent sphere radii } & $R_{\mathrm{v}}$ & $90 \mathrm{~nm}$ & $325 \mathrm{~nm}$ \\
& $R s$ & $190 \mathrm{~nm}$ & $1308 \mathrm{~nm}$ \\
& $R_{\mathrm{g}}$ & $211 \mathrm{~nm}$ & $591 \mathrm{~nm}$ \\
\hline
\end{tabular}

the degree of anisotropy of the aggregates [44]. In addition, it was shown that the equivalent-sphere model of hexagonal ice crystals underestimates the broadband solar albedo of cirrus clouds because of the sphere's stronger forward scattering compared to hexagonal crystals [45].

Let us define the different equivalent-sphere radii for fractal aggregates. Hereafter, the results using SDDA will be referred as SFA, whereas those using the equivalent-sphere approximation will be referred as $R_{v}, R_{S}, R_{g}$, respectively, depending on whether the equivalent volume, sphere, or gyration radii are used. The different equivalent sphere radii are given in Table 2.

$$
\begin{aligned}
& R_{v}=(N)^{\frac{1}{3}} R_{m} \\
& R_{s}=(N)^{\frac{1}{2}} R_{m} \\
& R_{r}=\left(\frac{N}{k_{f}}\right)^{\frac{1}{D_{f}}} R_{m}
\end{aligned}
$$

In the following, the extinction and backscattering crosssections for the equivalent-sphere approximations were computed with the Lorenz-Mie theory using the MIEV0 code [46] using the different equivalent radii displayed in Table 2.

\subsection{Extinction cross-sections}

In this section, extinction cross-sections were calculated using the SDDA model for SFA and Lorenz-Mie theory for the associated equivalent-spherical models. The same spectral complex refractive index was used as that in Section 2.2.

For the chain-like aggregate (Fig. 3), numerical computation with $R_{S}$ or $R_{g}$ leads to an overestimation of the spectral extinction cross-section computed with SFA for the complete spectrum. The value of $C_{\text {ext }}$ computed at $\lambda=800 \mathrm{~nm}$ for the SFA is approximately ten times smaller than that computed under the $R_{S}$ and $R_{g}$ equivalent-sphere assumptions. Regarding the spectral extinction cross-sections computed with $R_{v}$, this approximation provides results in close agreement with the SFA results.

For the compact aggregate (Fig. 4), the spectral extinction crosssection computed with $R_{S}$ or $R_{g}$ do not exhibit significant varia- 


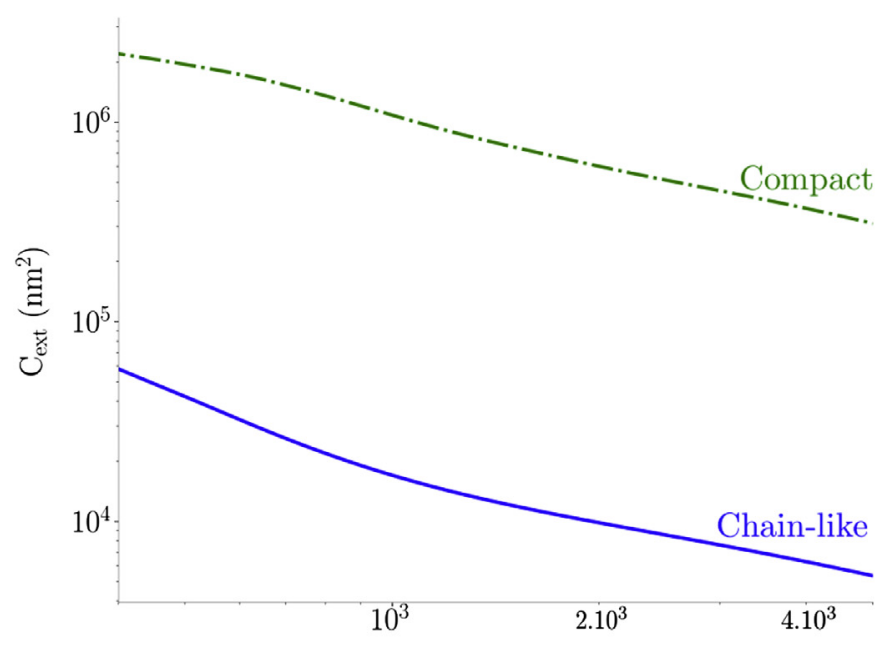

Wavelength $(\mathrm{nm})$

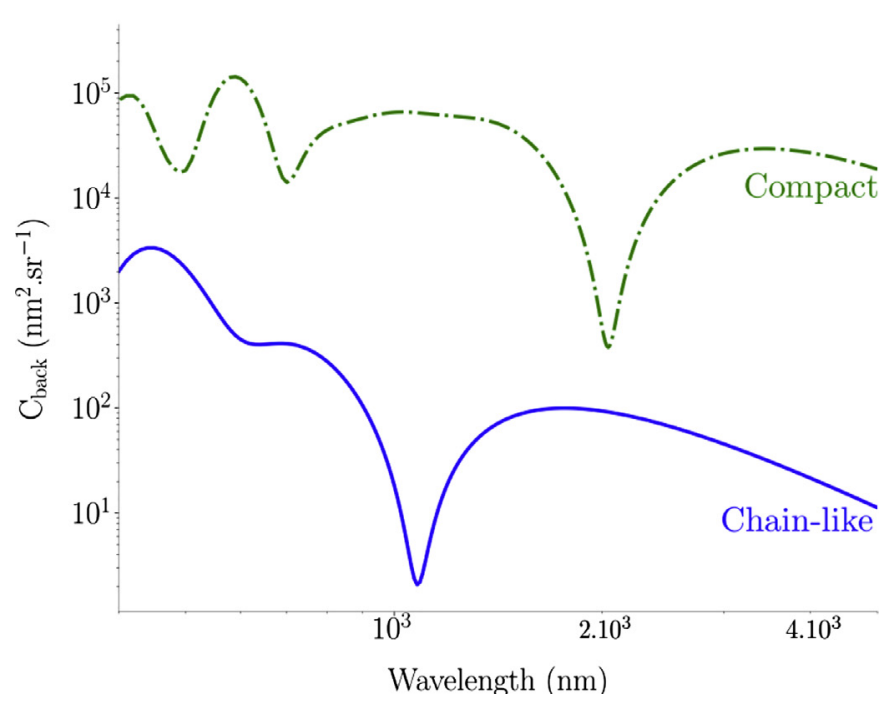

Fig. 2. Extinction and backscattering cross sections, $C_{\text {ext }}$ and $C_{\text {back }}$ respectively, for the chain-like (blue, continuous line) and compact (green, dashed line) aggregate are represented by continuous line dash lines, respectively.

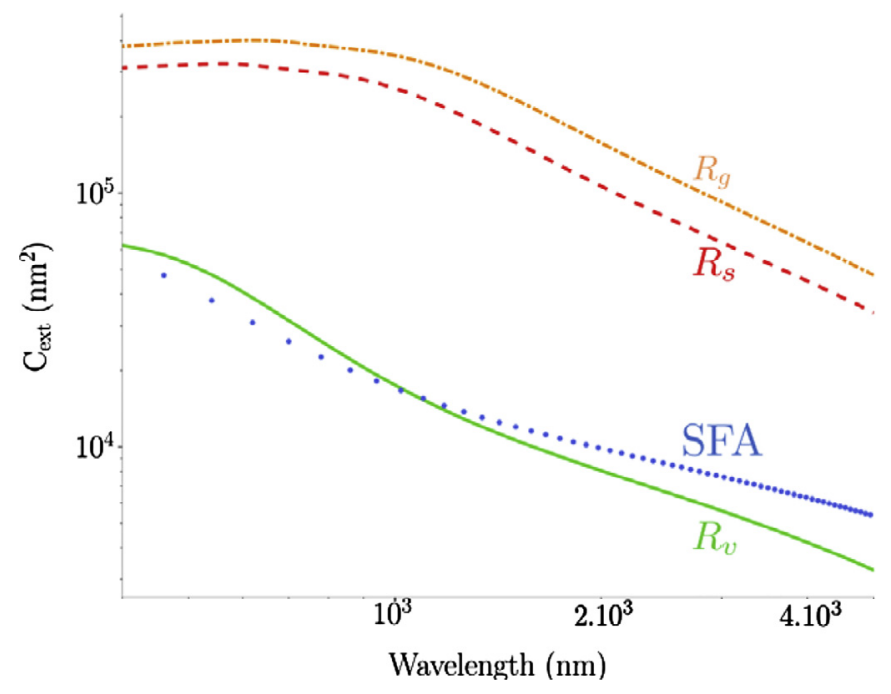

Fig. 3. Extinction cross-sections $C_{\text {ext }}$ of chain-like aggregate using fractal aggregate geometry (SFA, blue dots), volume $\left(R_{v}\right.$, green solid line), surface $\left(R_{s}\right.$, red dashed line), and gyration ( $R_{g}$, orange dash-dotted line) equivalent radii.

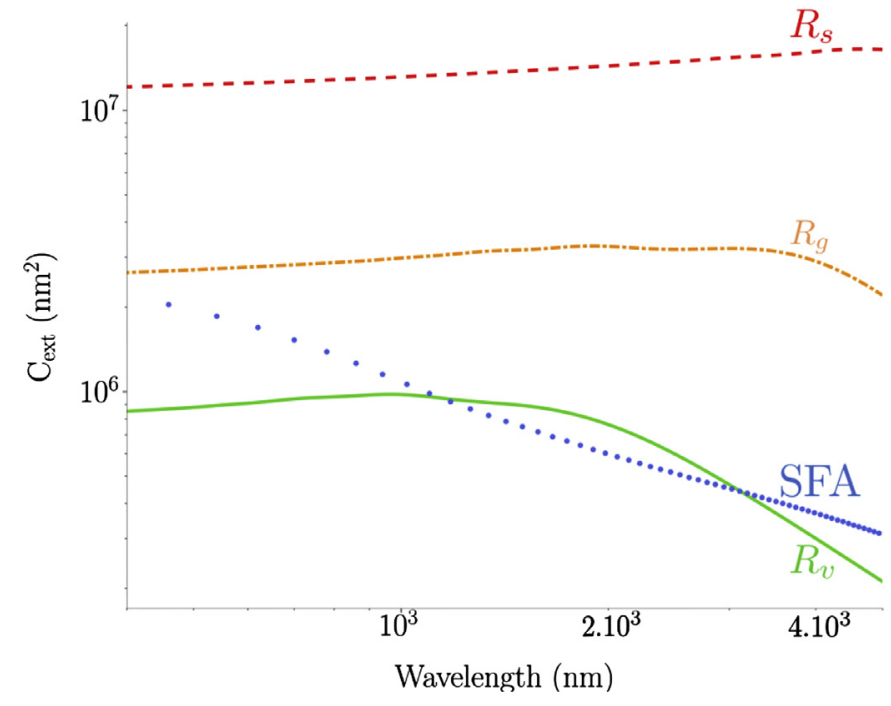

Fig. 4. Extinction cross-sections $C_{e x t}$ of compact aggregate using fractal aggregate geometry (SFA, blue dots), volume (Rv, green solid line), surface (Rs, red dashed line), and gyration ( $\mathrm{Rg}$, orange dash-dotted line) equivalent radii.

tions with $\lambda$ over $400-5000 \mathrm{~nm}$ unlike the chain-like aggregate. Also note that the results for $R_{g}$ overestimate those associated with SFA. For $\lambda<800 \mathrm{~nm}$, the spectral extinction cross-sections computed with $R_{v}$ and $R_{g}$ are in close agreement with the SFA results. This agreement strongly varies with the considered wavelength. Different results are obtained in the large-wavelength region for $\lambda>800 \mathrm{~nm}$ where the extinction cross-sections are better represented by $R_{v}$.

Overall, no sphere approximation can correctly describe the extinction cross-sections over the broadband spectrum. Only the volume equivalent-sphere approximation $\left(R_{v}\right)$ comes close, and only in the infrared part of the spectrum. This behavior is explained by the fact that soot exhibits strong absorption in the infrared, which is directly related to the total volume of the aggregate [47]. As a result, when absorption dominates in extinction, the volume equivalent-sphere approximation provides the best result of the sphere surrogates considered. In the visible however, where SFAs exhibit both absorption and scattering, equivalent-sphere approximations should be used with great caution for these two studied SFA.

\subsection{Backscattering cross-sections}

In this section, backscattering cross-sections were calculated using the SDDA model for SFAs and Lorenz-Mie theory for the associated equivalent spheres. The same spectral complex refractive index was used as that in Section 2.2.

For the chain-like aggregate (Fig. 5), Mie oscillations are shown from $400-2000 \mathrm{~nm}$ for the $R_{S}$ and $R_{g}$ approximations. These oscillations occur at different wavelengths depending on the equivalentradius to wavelength parameter. The backscattering cross-sections computed under the $R_{S}$ and $R_{g}$ approximations are close to the results computed by SFA for small wavelength $(\lambda<1000 \mathrm{~nm})$. The results using the $R_{S}$ and $R_{g}$ approximations remain twice as large as the SFA results for large wavelengths $(\lambda>1000 \mathrm{~nm})$. Only the $R_{v}$ approximation is in agreement with the SFA results for large wavelength in the infrared.

For the compact aggregate (Fig. 6), Mie oscillations also appear from $400-5000 \mathrm{~nm}$ for all approximations schemes. This leads to significant variations of the spectral extinction cross-section under the equivalent sphere approximation from the SFA results. It is worth noting that the results associated with $R_{S}$ and $R_{g}$ overestimate the SFA backscattering cross-sections. Yet, between 3000- 


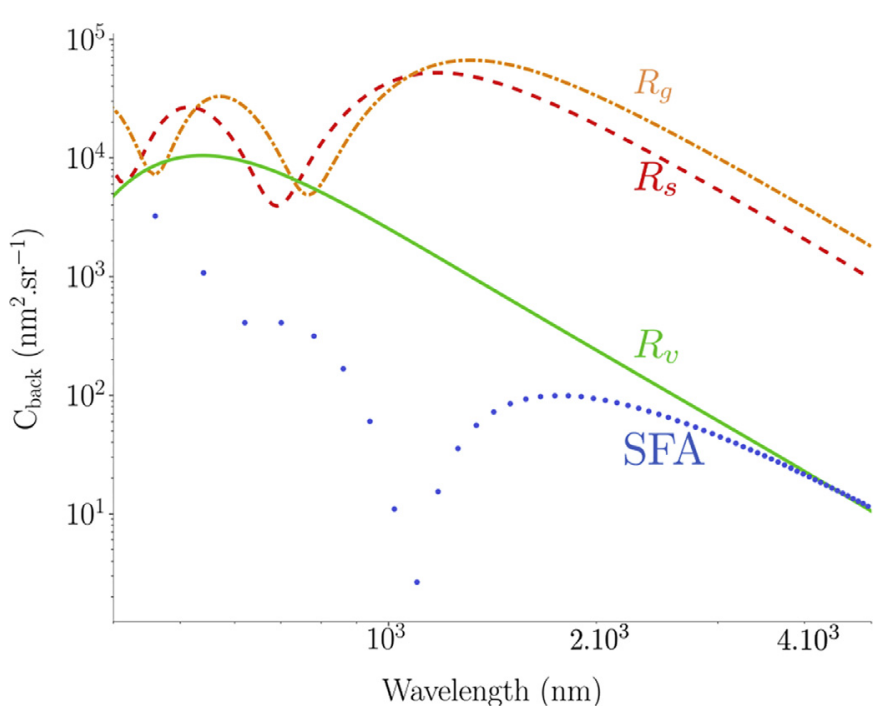

Fig. 5. Backscattering cross-sections $C_{\text {back }}$ of chain-like aggregate using fractal aggregate geometry (blue dots) for volume $\left(R_{v}\right.$, green solid line), surface $\left(R_{s}\right.$, red dashed line), and gyration ( $R_{g}$, orange dash-dotted line) equivalent radii.

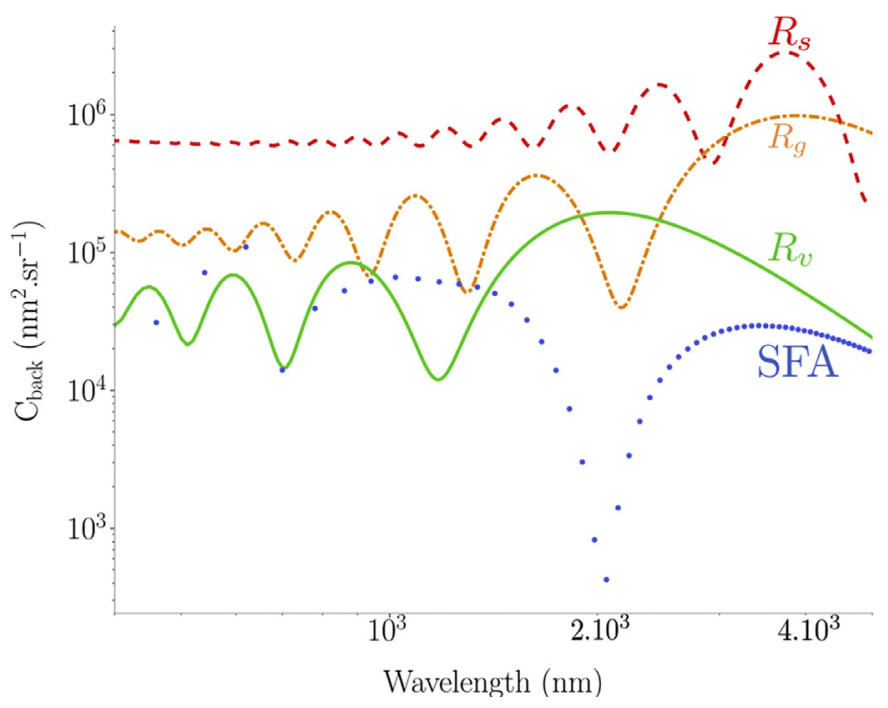

Fig. 6. Backscattering cross-sections $C_{\text {back }}$ of compact aggregate using fractal aggregate geometry (SFA, blue dots) for volume $\left(R_{v}\right.$, green solid line), surface $\left(R_{s}\right.$, red dashed line), and gyration ( $R_{g}$, orange dash-dotted line) equivalent radii.

$5000 \mathrm{~nm}$, the spectral backscattering cross-sections computed with $R_{v}$ are in close agreement with the SFA results. Different results are obtained for $\lambda<3000 \mathrm{~nm}$ where no equivalent sphere radius approximates the SFA backscattering cross-sections.

In summary, the equivalent-sphere approximation gives poor results in terms of computing the backscattering cross-sections for the SFAs considered. Only $R_{v}$ approximation is acceptable for $\lambda>$ $4000 \mathrm{~nm}$. Hence, according to our simulations, the $C_{\text {back }}$ is more sensitive than $C_{\text {ext }}$ to the shape of the aggregate considered than the extinction cross-section. This is explained because $C_{\text {back }}$ corresponds to the differential scattering cross-sections at $180^{\circ}$ whereas $C_{\text {ext }}$ is an angularly integrated quantity.

\section{Discussion}

From our results, cross-sections relevant to lidar, i.e. extinction and backscattering, appear to strongly depend on the morphology of the SFAs. Although approximations often provide valu- able insight into physical processes in light-scattering, our results highlight that the concept of the equivalent-sphere approximation should be used carefully, only over restricted spectral ranges. Moreover, the choice of equivalent radii clearly impacts, in a different manner, both the extinction and backscattering cross sections.

The extinction cross-section refers to the reduction of the power received by a detector facing the incident wave when a particle is present in the optical path compared to when the particle is absent. Thus, the volume is expected to be the major factor contributing to the extinction cross-section of a small particle when absorption is important. The key for computing the extinction cross-section under the equivalent sphere approximation is to choose the right equivalent radii to reproduce the correct scattering quantity. Our results show that as long as the volume of the aggregate is conserved, the volume equivalent sphere is a better approximation to compute the extinction cross-section, especially in the infrared where the absorption of soot is high, whatever the fractal-like geometry, i.e. chain-like or compact.

However, the equivalent-spherical models may be more difficult to apply to compute the backscattering cross-sections of SFAs. The latter refers to the backscattering part of the differential scattering cross-section, which highly depends on the geometry of the particle [48]. As the particle geometry largely affects the differential scattering cross-section [32], the backscattering cross-sections is expected to depend sensitively on the detailed geometry of the aggregate. Our results highlight that equivalent-sphere models fail to reproduce the lidar cross-sections over a broadband spectrum. Obviously, substituting a fractal-like aggregate for a sphere is an extreme modification of the original-particle shape, substantially changing the scattered electromagnetic fields, and thus, the cross sections.

A general feature can be highlighted: among the different equivalent radii that can be used to approximate the lidar crosssections, the equivalent volume radius $\left(R_{v}\right)$ seems to be a much better parameter, especially for large wavelengths where absorption is important. On the whole, using equivalent surface or gyration radius $\left(R_{S}\right.$ or $R_{g}$ ) leads to overestimation, by several orders of magnitude, of both extinction and backscattering cross sections regardless of the fractal dimension. This overestimation is seen for most wavelengths in the range considered, especially small wavelengths. Such behavior can be explained by the ratio between the aggregate size and the wavelength. At large wavelength, interaction with the incident wave occurs largely without sensing the fractal details of aggregates: the whole aggregate scatters as a volume equivalent-sphere. Conversely, interaction at short wavelength does sense the detailed aggregate structure. The morphology of aggregates thus has a strong impact on the lidar crosssections when the wavelength approaches the size of aggregate as one would expect. This explains why there is no appropriate equivalent sphere approximation for soot fractal aggregates at small wavelength.

The equivalent-sphere model applied to fractal aggregates has a highly limited capacity for calculating lidar cross-sections, especially backscattering. Furthermore, no equivalent-sphere approximation should be used as a substitute for broadband lidar crosssections. This is particularly true for backscattering cross-sections at small and intermediate wavelengths (e.g. near UV and visible) where scattering is important. In the infrared, one may consider the volume equivalent-sphere approximation for backscattering cross-sections. These results remain valid for SFAs with comparable sizes however, for larger aggregates the equivalent spherical model should be used even more cautiously. The negative consequences for lidar inversion techniques are likely beyond the scope of this work, but one should use great care when implementing them in lidar aerosol-retrievals. 


\section{Conclusion}

The ability of different equivalent-sphere models to reproduce the extinction and backscattering cross sections of soot aggregates was investigated over a broadband range of wavelengths (400$5000 \mathrm{~nm}$ ). A set of numerical computations were performed with a spectral DDA model on a chain-like and compact soot aggregates to assess the validity of these approximations. This is of crucial importance in the field of lidar remote sensing and retrieval of aerosol microphysical parameters from lidar signals. It has been shown that the volume-equivalent spherical radius is a quite good parameter to represent the extinction cross section of soot aggregates, over the majority of the spectral range considered. On the contrary, the backscattering cross section is much more sensitive to the value of the size parameter. These results could have significant implications on the development of inversion methods involving soot aggregates.

\section{Acknowledgements}

This research was partially funded by the PROMETE project. This work has been conducted in the framework of the collaboration agreement between Kansas State University and ONERA, The French Aerospace Lab.

\section{References}

[1] Bond TC, Doherty SJ, Fahey DW, Forster PM, Berntsen T, et al. Bounding the role of black carbon in the climate system: a scientific assessment. J Geophys Res Atmos 2013;118(11):5380-552. doi:10.1002/jgrd.50171.

[2] Bockhorn H. Soot formation in combustion mechanisms and models. Berlin: Springer-Verlag; 1994.

[3] Calvo AI, Alves C, Castro A, Pont V, Vicente AM, Fraile R. Research on aerosol sources and chemical composition: past, current and emerging issues. Atmos Res 2013;120:1-28 ISSN 0169-8095.

[4] Samson RJ, Mulholland GW, Gentry JW. Langmuir 1987;3(2):272-81. doi:10. 1021/la00074a022.

[5] Dobbins RA, Megaridis CM. Morphology of flame-generated soot as determined by thermophoretic sampling. Langmuir 1987;3:254-9. doi:10.1021/ la00074a019.

[6] Köylü ÜÖ, Faeth GM, Farias TL, Carvalho MG. Fractal and projected structure properties of soot aggregates. Combust Flame 1995;100(4):621-33. doi:10. 1016/0010-2180(94)00147-K.

[7] Dastanpour R, Rogak SN. Observations of a correlation between primary particle and aggregate size for soot particles. Aerosol Sci Technol 2014;48(10):10439. doi:10.1080/02786826.2014.955565.

[8] Liu F, Wong C, Snelling DR, Smallwood GJ. Investigation of absorption and scattering properties of soot aggregates of different fractal dimension at $532 \mathrm{~nm}$ using RDG and GMM. Aerosol Sci Technol 2013;47(12). doi:10.1080/02786826. 2013.847525.

[9] Doner N, Liu F. Impact of morphology on the radiative properties of fractal soot aggregates. J Quant Spectroscop Rad Transf 2017;187:10-19 ISSN 00224073. doi:10.1016/j.jqsrt.2016.09.005.

[10] Mansurov ZA. Soot formation in combustion processes (review). Combustion. Explosion Shock Waves 2005;41(6):727-44. doi:10.1007/s10573-005-0083-2.

[11] Ramanathan V, Carmichael G. Global and regional climate changes due to black carbon. Nat Geosci 2008;1(4):221-7. doi:10.1038/ngeo156.

[12] Popovicheva OB, Persiantseva NM, Lukhovitskaya EE, Shonija NK, Zubareva NA, Demirdjian B, Ferry D, Suzanne J. Aircraft engine soot as contrail nuclei. Geophys Res Lett 2004;31(11). doi:10.1029/2003GL018888.

[13] Kärcher B, Yu F. Role of aircraft soot emissions in contrail formation. Geophys Res Lett 2009;36(L01804):1-5. doi:10.1029/2008GL036649.

[14] Forget F, Pierrehumbert RT. Warming early Mars with carbon dioxide clouds that scatter infrared radiation. Science 1997;278(5341):1273-6. doi:10.1126/ science.278.5341.1273.

[15] Stocker TF, Qin D, Plattner GK, Tignor M, Allen SK, Boschung J, Nauels A, Xia Y, Bex V, Midgley PM. Climate change 2013: the physical science basis. contribution of working group I to the fifth assessment report of the intergovernmental panel on climate change. IPCC., 1535. Cambridge, United Kingdom and New York, NY, USA: Cambridge University Press; 2013. doi:10.1017/ CBO9781107415324.

[16] Boyland E. The toxicology of soot. Soot in combustion systems and its toxic properties NATO conference series (VI materials science), 7 Boston, MA. Springer; 1983. doi:101007/978-1-4684-4463-6_2.

[17] Lighty JS, Veranth JM, Sarofim AF. Combustion aerosols: factors governing their size and composition and implications to human health. J Air Waste Manag Assoc 2000;50(9):1565-618 PMID: 11055157.

[18] Su DS, Serafino A, Müller JO, Jentoft RE, Schlögl R, Fiorito S. Cytotoxicity and inflammatory potential of soot particles of low-emission diesel engines. Environ Sci Technol 2008;42(5):1761-5. doi:10.1021/es071655.
[19] Lidar CollisRTH. A new atmospheric probe. QJR Meteorol Soc 1966;92:220-30. doi:10.1002/qj.49709239205.

[20] Measures RM. Laser remote sensing: fundamentals and applications. John Wiley \& Sons; 1992.

[21] Heintzenberg J, Müller H, Quenzel H, Thomalla E. Information content of optical data with respect to aerosol properties: Numerical studies with a randomized minimization-search-technique inversion algorithm. Applied Opt 1981;20(8):1308-15. doi:10.1364/A0.20.001308.

[22] Sasano Y, Browell EV. Light scattering characteristics of various aerosol types derived from multiple wavelength lidar observations. Appl Opt 1989;28(9):1670-9. doi:10.1364/AO.28.001670.

[23] Feingold G, Grund CJ. Feasibility of using multiwavelength lidar measurements to measure cloud condensation nuclei. J Atmos Ocean Technol 1994;11:154358 doi:10.1175/1520-0426(1994)011〈1543:FOUMLM〉2.0.CO;2.

[24] Althausen D, Müller D, Ansmann A, Wandinger U, Hube H, Clauder E, Zörner S. Scanning six-wavelength eleven-channel aerosol lidar. J Atmos Oceanic Technol 2000;17:1469-82 doi:10.1175/1520-0426(2000)017〈1469:SWCAL〉2.0.CO;2.

[25] Wandinger U, Müller D, Böckmann C, Dietrich A, Volker M, et al. Optical and microphysical characterization of biomass-burning and industrial-pollution aerosols from multiwavelength lidar and aircraft measurements. J Geophys Res 2002;107(D21). doi:10.1029/2000JD000202,2002.

[26] Müller D, Wagner F, Wandinger U, Ansmann A, Wendisch M, Althausen D, von Hoyningen-Huene W. Microphysical particle parameters from extinction and backscatter lidar data by inversion with regularization: experiment. Appl Opt 2000;39(12):1879-92. doi:10.1364/A0.39.001879.

[27] Veselovskii I, Kolgotin A, Müller D, Whiteman DN. Information content of multiwavelength lidar data with respect to microphysical particle properties derived from eigenvalue analysis. Appl Opt 2005;44(25):5292-303. doi:10.1364/ A0.44.00529.

[28] Burton SP, Hair JW, Kahnert M, Ferrare RA, Hostetler CA, Cook A, et al. Observations of the spectral dependence of linear particle depolarization ratio of aerosols using NASA langley airborne high spectral resolution lidar. Atmos Chem Phys 2015;15:13453-73. doi:10.5194/acp-15-13453-2015.

[29] Mishchenko MI, Dlugach JM, Liu L. Linear depolarization of lidar returns by aged smoke particles. Appl Opt 2016;55(35):9968-73. doi:10.1364/AO.55. 009968.

[30] Mishchenko MI, Liu L, Mackowski DW. T-matrix modeling of linear depolarization by morphologically complex soot and soot-containing aerosols. J Quant Spectrosc Radiat Transfer 2013;123:135-44. doi:10.1016/j.jqsrt.2012.11.012.

[31] Donovan DP, Quante M, Schlimme I, Macke A. Use of equivalent spheres to model the relation between radar reflectivity and optical extinction of ice cloud particles. Appl Opt 2004;43(25):4929-40. doi:10.1364/A0.43.004929.

[32] Li H, Liu C, Bi L, Yang P, Kattawar GW. Numerical accuracy of "equivalent" spherical approximations for computing ensemble-averaged scattering properties of fractal soot aggregates. J Quant Spectrosc Radiat Transf. 2010;111(14):2127-32. doi:10.1016/j.jqsrt.2010.05.009.

[33] Ceolato R, Berg MJ, Riviere N. Spectral and angular light-scattering from silica fractal aggregates. J Quant Spectrosc Radiat Transf 2013;131:160-5. doi:10. 1016/j.jqsrt.2013.01.007.

[34] Jullien R, Botet R. Aggregation and fractal aggregates. Singapore: World Scientific; 1987

[35] Meakin P. Formation of fractal clusters and networks by irreversible diffusion-limited aggregation. Phys Rev Lett 1983;51:1119-22.

[36] Kolb M, Botet R, Jullien R. Scaling of kinetically growing clusters. Phys Rev Lett 1983;51:1123-6. doi:10.1103/PhysRevLett.51.1123.

[37] Botet R, Jullien R, Kolb M. Hierarchical model for irreversible kinetic cluster formation. J Phys A 1984;17:L75-9.

[38] Purcell EM, Pennypacker CR. Scattering and absorption of light by nonspherical dielectric grains. Astrophys J 1973;186:705-14. doi:10.1086/152538.

[39] Yurkin MA, Hoekstra AG. The discrete dipole approximation: an overview and recent developments. J Quant Spectrosc Radiat Transf 2007;106(1):558-89. doi:10.1016/j.jqsrt.2007.01.034.

[40] Lallich S, Enguehard F, Baillis D. Experimental determination and modeling of the radiative properties of silica nanoporous matrices. J Heat Transf 2009;131(8) 082701-082701-12. doi:10.1115/1.3109999.

[41] Chang H, Charalampopoulos TT. Determination of the wavelength dependence of refractive indices of flame soot. Proc R Soc Lond A 1990;430:577-91. doi:10. 1098/rspa.1990.0107.

[42] Draine BT, Flatau PJ. Discrete-dipole approximation for scattering calculations. J Opt Soc Am A 1994;11(4):1491-9. doi:10.1364/JOSAA.11.001491.

[43] Sorensen CM. Light scattering by fractal aggregates: a review. Aerosol Sci Technol 2001;35(2):648-87. doi:10.1080/02786820117868.

[44] Stephens G. Radiative properties of cirrus clouds in the infrared region. J Atm Sci 1980;37:435-46 doi:10.1175/1520-0469(1980)037/0435:RPOCCI 2.0.CO;2.

[45] Liou KN, Takano Y. Light scattering by nonspherical particles: remote sensing and climatic implications. Atmos Res 1994;31(4):271-98. doi:10.1016 0169-8095(94)90004-3.

[46] Wiscombe WJ. Mie scattering calculations: advances in technique and fast, vector-speed computer codes. NCAR/TN-140+STR. national center for atmospheric research Boulder, Colorado; 1979. doi:10.5065/D6ZP4414.

[47] Bohren CF, Huffman DR. Absorption and scattering of light by small particles. New York: Wiley; 1983. doi:10.1002/9783527618156.

[48] Yang P, Wei H, Kattawar GW, Hu YX, Winker DM, Hostetler CA, Baum BA. Sensitivity of the backscattering Mueller matrix to particle shape and thermodynamic phase. Appl Opt 2003;42(21):4389-95. doi:10.1364/AO.42.004389. 